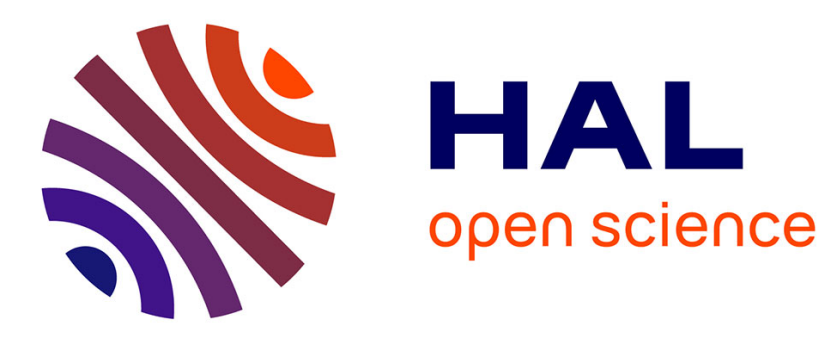

\title{
Increases in risk and demand for risky asset
}

Alain Chateauneuf, Ghizlane Lakhnati

\section{To cite this version:}

Alain Chateauneuf, Ghizlane Lakhnati. Increases in risk and demand for risky asset. 2005. halshs00194413

\section{HAL Id: halshs-00194413 \\ https://shs.hal.science/halshs-00194413}

Submitted on 6 Dec 2007

HAL is a multi-disciplinary open access archive for the deposit and dissemination of scientific research documents, whether they are published or not. The documents may come from teaching and research institutions in France or abroad, or from public or private research centers.
L'archive ouverte pluridisciplinaire HAL, est destinée au dépôt et à la diffusion de documents scientifiques de niveau recherche, publiés ou non, émanant des établissements d'enseignement et de recherche français ou étrangers, des laboratoires publics ou privés. 

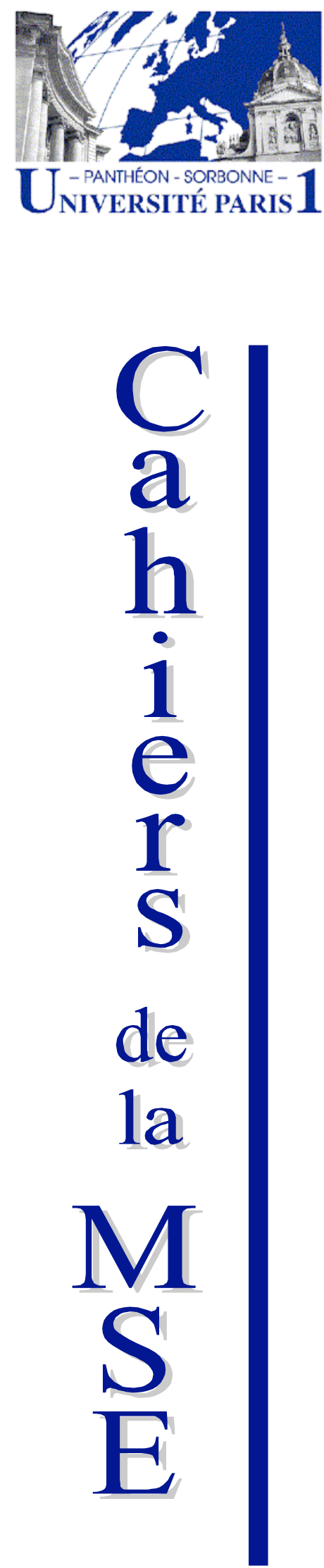

Increases in risk and demand for risky asset

Alain Chateauneuf, Cermsem

Ghizlane LAKHNATI, CERMSEM

2005.33 


\title{
Increases In Risk and Demand For Risky Asset
}

\author{
Alain Chateauneuf* Ghizlane Lakhnati ${ }^{\dagger}$
}

\begin{abstract}
Résumé
Dans ce papier nous examinons l'effet d'une diminution du risque sur la demande optimale en actif risqué dans le problème du choix de portefeuille standard. Nous introduisons une nouvelle classe de dominance, que nous appelons la dominance relative et nous montrons que cette nouvelle classe de dominance est compatible avec la dominance centrale introduite par Gollier [5] et l'accroissement du risque préservant la moyenne. Enfin, nous vérifions que certaines classes de dominances déjà existantes dans la littérature sont des cas particuliers de notre nouvelle dominance.
\end{abstract}

Mots Clés: Modèle EU, Choix de portefeuille, MPIR, Dominance Centrale, Dominance Simple Relative, Dominance Relative.

Classification JEL: D80, D81, G11.

\begin{abstract}
In this paper, we examine the effect of a decrease in risk on the demand for risky asset in the standard portfolio problem. We introduce a new class of dominance, that we name relative order and we prove that this class of dominance is consistent both with central dominance introduced by Gollier [5] and with mean preserving increase in risk. Finally, we show that some known classes of dominance are particular cases of our new class of dominance.
\end{abstract}

Keywords: EU Model, Portfolio Choice, Mean Preserving Increase in Risk, Central Dominance, Relative Simple Dominance, Relative Dominance.

JEL Classification: D80, G11.

${ }^{*}$ CERMSEM-Université de Paris 1, 106-112 Boulevard de l'Hôpital, 75647 Paris Cedex 13, France. E-mail: Chateauneuf@univ-paris1.fr

${ }^{\dagger}$ CERMSEM-Université de Paris 1, 106-112 Boulevard de l'Hôpital, 75647 Paris Cedex 13, France. E-mail: Lakhnati@univ-paris1.fr 


\section{Introduction}

Many authors have examined the comparative statics effect of a change in risk. It is known that second order stochastic dominance (SSD) is neither necessary nor sufficient to decrease the agent's demand for the risky asset after a shift in the risky asset, in the standard portfolio problem with a risk-free asset and a risky asset.

Gollier [5] characterized the necessary and sufficient condition on the change in the risky asset to guarantee that all risk averse agents will increase their demand of the risky asset. This condition is called Central Dominance (thereafter CD). In this paper we focus on changes in risk which preserve the mean and are in the intersection of SSD and CD.

The paper is organized as follows: In section 2 and 3, we present the framework, the decision model and we recall some important and preliminary results.

Rotschild and Stiglitz [12], Machina and Pratt [5] have shown that a mean preserving increase in risk (MPIR) can be obtained by adding a noise to the less risky random variable, or by a sequence of one or more mean preserving spreads (thereafter MPS). In section 4, we propose a new class of dominance which is consistent with MPIR and CD. We start by a definition of a new dominance that we name relative simple dominance (thereafter RSD) and we prove that relative dominance is in the intersection set of CD and MPS. Hence we introduce our new dominance in its full generality, that we name relative dominance (thereafter $\mathrm{RD}$ ) and we prove that $\mathrm{RD}$ implies mean preserving spread and central dominance. Finally, we show that some classes of dominance already existing in the literature are particular cases of our dominance class: On one hand, we show that SIR introduced by Meyer and Ormiston [7], [8] is a particular case of relative dominance, on the other hand, the same applied for Simple Dominance introduced by Dionne and Gollier [3]. Moreover, we show that monotone mean preserving spread about the origin introduced by Quiggin [10] implies simple dominance, hence relative dominance.

\section{The decision model}

We consider a decision maker (DM thereafter) endowed with a initial wealth $w$. The set $\mathbb{V}$ of such assets consisting of all bounded real random variables defined on a probability space $(S, \mathcal{A}, P)$ assumed to be sufficiently rich to generate any bounded real-valued random variable. $S$ the set of states of nature, $\mathcal{A}$ a $\sigma$-algebra of subsets of $S$ and $P$ a $\sigma$-additive non-atomic probability measure. Any $X \in \mathbb{V}$ is a (real) bounded random variable characterized by a probability distribution, with $F_{X}$ its cumulative distribution function (i.e.: $\left.F_{X}(t)=P(X \leq t), \forall t \in \mathbb{R}\right)$.

When $X$ is a finite discrete random variable, it will be denoted as:

$\mathcal{L}(X)=\left(x_{1}, p_{1} ; x_{2}, p_{2} ; \ldots ; x_{n}, p_{n}\right)$, with $p_{i} \geq 0, \sum_{i=1}^{n} p_{i}=1$, assuming that $x_{1} \leq x_{2} \leq \ldots \leq x_{n}$.

We consider a strictly risk averse expected utility (EU) decision maker with a Von Neumann-Morgenstern utility function $u: \mathbb{R} \longrightarrow \mathbb{R}$, twice continuously 
differentiable, strictly concave, increasing and such that $u^{\prime}(x)>0, \forall x \in \mathbb{R}$.

One of the classical and important model in economic theory is: The Standard Portfolio Problem (see Dionne and al [4] and Gollier [5]). The DM has to determine the optimal composition of his portfolio containing a risk-free and a risky asset. The return of the risk-free asset is $\rho$. The return of the risky asset is a random variable $X$. Hence the problem of the DM is to determine the optimal composition $(w-\alpha, \alpha)$ of his portfolio, where $w-\alpha$ is invested in the risk-free asset and $\alpha$ is invested in the risky asset.

Thus, the payoff function in the last period is:

$$
W(X, \alpha)=(w-\alpha)(1+\rho)+\alpha(1+X)=w(1+\rho)+\alpha(X-\rho) .
$$

To simplify the model, we suppose that the risk-free rate $\rho=0$.

Hence, the payoff function is

$$
W(X, \alpha)=w+\alpha X
$$

The DM chooses $\alpha$ to maximize:

$$
U_{X}(\alpha)=E u(w+\alpha X)=\int_{\mathbb{R}} u(w+\alpha x) d F_{X}(x)
$$

As Gollier [5], we restrict attention, to situations where the DM will invest a strictly positive amount $\alpha$ in the risky asset, more precisely when (I) has a unique solution $\alpha^{*}$ and $\alpha^{*}>0$.

For $X$ belonging to $\mathbb{V}$, we denote $\left[a_{X}, b_{X}\right]$ the support of $F_{X}$, and thus confine risky assets to belong to the subset $\mathbb{V}^{+}$of $\mathbb{V}$ defined by:

$$
\mathbb{V}^{+}=:\left\{X \in \mathbb{V} \mid a_{X}<0, b_{X}>0 \text { and } E(X)>0\right\} .
$$

$\mathbb{V}_{0}^{+}$will denote the subset of $\mathbb{V}^{+}$, containing only finite discrete random variables: $X \in \mathbb{V}_{0}^{+}$, if it can be written such that:

$L(X)=\left(x_{1}, p_{1} ; x_{2}, p_{2} ; \ldots ; x_{k}, p_{k} ; \ldots ; x_{n}, p_{n}\right)$ with $x_{1}<\ldots<x_{k} \leq 0<x_{k+1}<$ $\ldots<x_{n}, p_{i}>0, \sum_{i=1}^{n} p_{i}=1$ and $\sum_{i=1}^{n} x_{i} p_{i}>0$.

Assuming the additional Inada condition for $u$ : $\lim _{z \rightarrow+\infty} u^{\prime}(z)=0$, it is therefore straightforward to check that (I) has a unique solution $\alpha_{X}^{*}$ defined by:

$$
U_{X}^{\prime}\left(\alpha_{X}^{*}\right)=E\left(X u^{\prime}\left(w+\alpha_{X}^{*} X\right)\right)=\int x u^{\prime}\left(w+\alpha_{X}^{*} x\right) d F_{X}(x)=0,
$$

and that $\alpha_{X}^{*}>0$, since $U_{X}^{\prime}(0)=E\left(X u^{\prime}(w)\right)=E(X) \cdot u^{\prime}(w)>0$.

\section{Preliminary results}

\subsection{Central Dominance}

The objective of many reachers has to determine the effect of a change in risk on the optimal portfolio. 
The problem is to find conditions which guarantee that all risk averters agents will react to the less risky situation by increasing the demand for the asset, i.e: $\alpha_{X}^{*} \geq \alpha_{Y}^{*}$, after a decrease in risk from $Y$ to $X$.

Let us present the famous result of Gollier about a new dominance which guarantees that all risk averse expected utility agents increase their exposure after a shift in distribution.

Gollier [5] proposes the following definition:

Definition $3.1 X$ centrally dominates $Y$ if and only if there exists a real scalar $m$ such that $\int_{-\infty}^{t} x d F_{X}(x) \geq m \int_{-\infty}^{t} x d F_{Y}(x), \forall t \in \mathbb{R}$. It is denoted $X \succeq_{C D} Y$.

In the particular case of discrete random variables in $\mathbb{V}^{+}$, the definition 3.1 translates as follows:

Definition 3.2 Let $X, Y \in \mathbb{V}_{0}^{+}$such that $L(X)=\left(z_{1}, p_{1} ; z_{2}, p_{2} ; \ldots ; z_{n}, p_{n}\right)$ and $L(Y)=\left(z_{1}, q_{1} ; z_{2}, q_{2} ; \ldots ; z_{n}, q_{n}\right)$ with $z_{1}<z_{2}<\ldots<z_{n}, p_{i} \geq 0, q_{i} \geq 0$ and $\sum_{i=1}^{n} p_{i}=\sum_{i=1}^{n} q_{i}=1$. X centrally dominates $Y$ if and only if there exists a real scalar $m$ such that: $\sum_{i=1}^{j} z_{i} p_{i} \geq m \sum_{i=1}^{j} z_{i} q_{i}, \forall j$.

Gollier [5] proved the following seminal result:

Proposition $3.1 X \succeq_{C D} Y$ is a necessary and sufficient condition to guarantee that all risk-averse agents increase their optimal demand for the risky asset when the excess return undergoes a decrease in risk from $Y$ to $X$.

\subsection{Mean Preserving Increase in Risk and Mean Preserving Spread}

Rotschild and Stiglitz [11] gives the necessary and sufficient condition for $X$ to be preferred to $Y$ by all risk averse $E U$ decision makers:

Definition 3.3 $X$ dominates $Y$ in the sense of second order stochastic dominance $\left(X \succeq_{S S D} Y\right)$ if:

$$
\int_{-\infty}^{t} F_{X}(x) d x \leq \int_{-\infty}^{t} F_{Y}(x) d x, \forall t \in \mathbb{R}
$$

If $X$ dominates $Y$ in the sense of SSD and if the mean is kept constant, then $Y$ is said to be a Mean Preserving Increase in Risk (MPIR) of X, denoted $Y$ MPIR $X$. Equally $X$ is said to be a Mean Preserving Reduction in Risk (MPRR) of $Y$, denoted $X$ MPRR $Y$.

A Mean Preserving Spread (see Rotschild and Stiglitz [12], Diamond and Stiglitz [2], Machina and Pratt [6], Cohen [1]) is a particular case of a Mean Preserving Increase in Risk. Thus:

Definition 3.4 $Y$ is a Mean Preserving Spread (MPS) of $X$, if $E(Y)=E(X)$ and there exists $t_{0} \in \mathbb{R}$ such that: $F_{X}(t) \leq F_{Y}(t), \forall t<t_{0}$ and $F_{X}(t) \geq$ $F_{Y}(t), \forall t \geq t_{0}$. 
Remark 3.1 It is known (see Rotschild and Stiglitz [12], Machina and Pratt [6]) that $Y$ MPIR $X$ if and only if $Y$ can be obtained from $X$ by a sequence of mean preserving spreads; that is there exists a sequence $X_{0}=X, X_{1}, \ldots, X_{n}$ such that $X_{n+1}$ differs from $X_{n}$ by a mean preserving spread and $X_{n} \rightarrow^{d} Y{ }^{1}$

Now, we show by means of two examples that MPIR is not necessary nor sufficient for CD.

Counter-examples:

1) Sufficiency:

$L(X)=\left(-4, \frac{1}{8} ; 1, \frac{1}{16} ; 2, \frac{3}{16} ; 3, \frac{3}{16} ; 4, \frac{7}{16}\right)$ and $L(Y)=\left(-4, \frac{1}{8} ; 1, \frac{1}{8} ; 2, \frac{1}{8} ; 3, \frac{1}{8} ; 4, \frac{1}{2}\right)$.

We have that $Y M P I R X$, but $X$ does not centrally dominate $Y$.

2) Necessity:

$L(X)=\left(-1, \frac{1}{4} ; 1, \frac{1}{4} ; 3, \frac{1}{2}\right)$ and $L(Y)=\left(-1, \frac{1}{3} ; 1, \frac{1}{3} ; 5, \frac{1}{3}\right)$

$X \succeq_{C D} Y$ with $m=\frac{3}{4}$, but $X$ does not dominate $Y$ in the sense of MPIR, since $E(X)=\frac{3}{2}<E(Y)=\frac{5}{3}$.

Remark 3.2 It is important to notice that if $\int_{-\infty}^{t} x d F_{X}(x) \geq m \int_{-\infty}^{t} x d F_{Y}(x)$, $\forall t \in \mathbb{R}$ and $E(X)=E(Y)>0$, then $m$ must belong to the interval $(0,1]$.

Indeed:

Let $X$ and $Y$ in $\mathbb{V}^{+}$such that $X \succeq_{C D} Y$ and $\left[a_{1}, b_{1}\right],\left[a_{2}, b_{2}\right]$ be respectively the supports of $F_{X}, F_{Y}$. Take $b=\max \left(b_{1}, b_{2}\right)$.

If $t=b$, we obtain $E(X) \geq m E(Y)$. Since $E(X)=E(Y), m \leq 1$.

\section{Relative Dominance}

Our goal in this part of paper is to propose a new class of changes in risk which is consistent both with MPIR and CD.

\subsection{Relative Simple Dominance}

Now, we concentrate on a new class of dominance, that will be sufficient to get central dominance with $m=1$ and mean preserving spread.

Definition 4.5 $X, Y \in \mathbb{V}^{+}, X$ is less risky then $Y$ in the sense of relative simple dominance (denoted $X \succeq_{R S D} Y$ ) if: $E(X)=E(Y)$ and there exists $t_{0}$ such that:

1. $F_{X}(t) \leq F_{Y}(t), \quad \forall t<t_{0}$.

2. $F_{X}(t) \geq F_{Y}(t), \quad \forall t \geq t_{0}$.

3. $F_{X}(t)-F_{Y}(t)$ is non-decreasing on $\left[t_{0}, 0\right)$, if $t_{0}<0$ $F_{X}(t)-F_{Y}(t)$ is non-decreasing on $\left[0, t_{0}\right)$, if $t_{0}>0$

\footnotetext{
${ }^{1}$ A sequence $\left(X_{n}\right)_{n}$ in $\mathbb{V}$ converges in distribution to $X$, denoted by $X_{n} \rightarrow{ }^{d} X$, if the sequence of distribution functions $F_{X_{n}}$ converges to distribution function $F_{X}$ at every continuity point of the latter.
} 
This definition simply says that $F_{X}$ crosses $F_{Y}$ once from below at $t_{0}$ and the difference between the final distribution function and the initial distribution function is non-decreasing in the left-closed interval with end-points 0 and $t_{0}$. In the particular case of discrete random variables in $\mathbb{V}^{+}$, the definition 4.3 translates as follows:

Definition 4.6 Let $X, Y \in \mathbb{V}_{0}^{+}$such that $L(X)=\left(z_{1}, p_{1} ; z_{2}, p_{2} ; \ldots ; z_{k}, p_{k} ; \ldots ; z_{n}, p_{n}\right)$ and $L(Y)=\left(z_{1}, q_{1} ; z_{2}, q_{2} ; \ldots ; z_{k}, q_{k} ; \ldots ; z_{n}, q_{n}\right)$ with $z_{1}<z_{2}<\ldots<z_{k} \leq 0<$ $z_{k+1}<\ldots<z_{n}, q_{i}+\epsilon_{i}=p_{i}, \sum_{i=1}^{n} \epsilon_{i}=0$.

$X$ is less risky then $Y$ in the sense of relative simple dominance if $\sum_{i=1}^{n} z_{i} \epsilon_{i}=0$ and there exists $j \geq 2$ such that:

1. $\forall r \in\{1, \ldots, j-1\}, \quad \sum_{i=1}^{r} \epsilon_{i} \leq 0$.

2. $\forall r \in\{j, \ldots \ldots \ldots, n\}, \quad \sum_{i=1}^{r} \epsilon_{i} \geq 0$.

3. If $j \leq k \quad \epsilon_{r} \geq 0, \quad \forall r \in\{j, \ldots, k\}$.

If $j \geq k+1 \quad \epsilon_{r} \geq 0, \quad \forall r \in\{k+1, \ldots, j\}$.

The following lemma recalls the integration by parts formula, which will prove useful for dealing with general distribution functions.

Lemma 4.1 Suppose that $G$ and $H$ are of bounded variations over the interval $[a, b] \subset \mathbb{R}$, then:

$$
\int_{[a, b]} H\left(t^{+}\right) d G(t)+\int_{[a, b]} G\left(t^{-}\right) d H(t)=H\left(b^{+}\right) G\left(b^{+}\right)-H\left(a^{-}\right) G\left(a^{-}\right) .
$$

Particulary, if $G(t)=t$ and $H$ is right continuous, we have:

$$
\int_{[a, b]} H(t) d t+\int_{[a, b]} t d H(t)=b H(b)-a H\left(a^{-}\right) .
$$

Proof: For sake of completeness a proof is given in appendix.

Theorem 4.1 Let $X, Y \in \mathbb{V}^{+}$, if $X \succeq_{R S D} Y$ then $Y M P S X$ and $X \succeq_{C D} Y$ with $m=1$.

Proof

That $Y M P S X$ is straightforward since $Y$ is a Mean Preserving Spread of $X$.

Let us now prove that $X \succeq_{C D} Y$ with $m=1$.

Let $\left[a_{1}, b_{1}\right]$ and $\left[a_{2}, b_{2}\right]$ be respectively the supports of $F_{X}$ and $F_{Y}$.

Take $a=\min \left(a_{1}, a_{2}\right)$ and $b=\max \left(b_{1}, b_{2}\right)$ and define $H(x)=F_{X}(x)-F_{Y}(x)$. We need to prove that $\delta(t)=\int_{-\infty}^{t} x d H(x)$ satisfies $\delta(t) \geq 0, \forall t \in \mathbb{R}$.

Note that since $H(x)=0, \forall x<a$ and $H(x)=0, \forall x>b$, we only need to prove that $\delta(t)=\int_{a}^{t} x d H(x) \geq 0, \forall t, a \leq t \leq b$.

Note also that $\delta(b)=0$, since $E(X)=E(Y)$ implies $\int_{a}^{b} x d H(x)=0$; from (4.1) since $H$ is right continuous and $H\left(a^{-}\right)=0$, this also implies that

$$
\int_{a}^{b} H(x) d x=0
$$


Finally from (4.1), we need to prove that for any $t \in[a, b], \delta(t)=t H(t)-$ $\int_{a}^{t} H(x) d x$ is non-negative.

Let us consider successively the cases when $t_{0} \leq 0$ and $t_{0}>0$.

Case 1: $t_{0} \leq 0$ :

Case 1.1: $t<t_{0}$ : Since $H(x) \leq 0, \forall x<t_{0}$, it comes that $\delta(t) \geq 0$.

Case 1.2: $t_{0} \leq t<0$ :

Note that if $t_{0}=0$, this case vanishes, so we assume that $t_{0}<0$.

From (4.2), $\delta(t)=t H(t)+\int_{t}^{b} H(x) d x$, hence: $\delta(t)=t H(t)+\int_{t}^{0} H(x) d x+$ $\int_{0}^{b} H(x) d x$.

From $H(x)$ non-decreasing on $[t, 0)$ comes $\int_{t}^{0} H(x) d x \geq-t H(t)$, hence $\delta(t) \geq$ $\int_{0}^{b} H(x) d x$, and $H(x) \geq 0, \forall x \geq 0$, gives $\delta(t) \geq 0$.

Case 1.3: $0 \leq t \leq b$ :

$\delta(t)=t H(t)+\int_{t}^{b} H(x) d x, H(x) \geq 0, \forall x \geq 0$, gives $\delta(t) \geq 0$.

Case 2: $t_{0}>0$ :

Case 2.1: $t \leq 0$ :

$\delta(t)=t H(t)-\int_{a}^{t} H(x) d x$. From $H(x) \leq 0, \forall x \leq 0$, it comes that $\delta(t) \geq 0$.

Case 2.2: $0<t<t_{0}$ :

$\delta(t)=t H(t)+\int_{a}^{0}(-H(x)) d x+\int_{0}^{t}(-H(x)) d x$.

$-H(x)$ non-increasing on $[0, t]$ implies $\int_{0}^{t}(-H(x)) d x \geq-t H(t)$, hence $\delta(t) \geq$ $\int_{a}^{0}(-H(x)) d x, H(x) \leq 0, \forall x \leq 0$ gives $\delta(t) \geq 0$.

Case 2.3: $t_{0} \leq t \leq b$ : $\delta(t)=t H(t)+\int_{t}^{\bar{b}} H(x) d x, H(x) \geq 0, \forall x \geq t_{0}$, implies $\delta(t) \geq 0$.

Which completes the proof.

Remark 4.3 Needless to say relative simple dominance is a sufficient condition for $C D$ but not a necessary one.

Counter-example:

Let $X$ and $Y$ be defined as:

$L(X)=\left(-1, \frac{1}{8} ; 1, \frac{1}{8} ; 2, \frac{3}{8} ; 6, \frac{3}{8}\right)$ and $L(Y)=\left(-1, \frac{1}{4} ; 1, \frac{1}{4} ; 6, \frac{1}{2}\right)$

$E(Y)=E(X)=3$. It is very easy to verify that: $X \succeq_{C D} Y$ with $m=1$, but $X$ does not dominate $Y$ in the sense of relative simple dominance.

\subsection{Relative Dominance}

Accordingly, we introduce our new dominance in its full generality:

Definition $4.7 X$ is less risky than $Y$ in the sense of relative dominance denoted $X \succeq_{R D} Y$, where $\succeq_{R D}$ is the transitive closure of $\succeq_{R S D}$. More precisely $X \succeq_{R D} Y$ if there exists a sequence of uniformly bounded $\left(X_{n}\right)_{n \in \mathbb{N}}, X_{n} \in \mathbb{V}^{+}$ 
such that $X_{0}=X, X_{n} \succeq_{R S D} X_{n+1}, \forall n \in \mathbb{N}$ and $X_{n}$ converges in distribution towards $Y$.

Example of relative dominance:

Let $X$ and $Y$ be defined as:

$L(X)=\left(-1, \frac{1}{16} ; 1, \frac{7}{16} ; 4, \frac{1}{2}\right)$ and $L(Y)=\left(-1, \frac{1}{4} ; 1, \frac{1}{4} ; 4, \frac{1}{4} ; 8, \frac{1}{4}\right)$

We have that $X \succeq_{R D} Y$, since:

$X_{0}=X \succeq_{R S D} X_{1}$, where $L\left(X_{1}\right)=\left(-1, \frac{3}{32} ; 1, \frac{6}{16} ; 4, \frac{5}{16} ; 8, \frac{7}{32}\right)$

$X_{1} \succeq_{R S D} X_{2}$, where $L\left(X_{2}\right)=\left(-1, \frac{1}{8} ; 1, \frac{5}{16} ; 4, \frac{3}{8} ; 8, \frac{3}{16}\right)$

and $X_{2} \succeq_{R S D} X_{3}=Y$.

Theorem 4.2 If $X \succeq_{R D} Y$ then $Y$ MPIR $X$ and $X \succeq_{C D} Y$ with $m=1$.

Proof

From Remark 3.1, Y MPIRX.

Moreover, from theorem $4.1 X_{n} \succeq_{C D} X_{n+1}$ with $m=1, \forall n \in \mathbb{N}$ i.e. $\int_{-\infty}^{t} x d F_{X_{n}}(x)$ $\geq \int_{-\infty}^{t} x d F_{X_{n+1}}(x), \forall t \in \mathbb{R}$. Hence $\int_{-\infty}^{t} x d F_{X}(x) \geq \int_{-\infty}^{t} x d F_{X_{n}}(x), \forall t \in \mathbb{R}$.

$X_{n}$ uniformly bounded, and $X_{n} \rightarrow^{d} Y$ implies $\int_{-\infty}^{t} x d F_{X} \geq \int_{-\infty}^{t} x d F_{Y}, \forall t \in \mathbb{R}$ i.e. $X \succeq_{C D} Y$ with $m=1$, which completes the proof.

\subsection{Strong Risk Dominance and Simple Dominance}

In this section, we show that some classes of dominance already existing in the literature can be derived from our new more general dominance.

\subsubsection{Strong Risk Dominance}

Meyer and Ormiston [7], [8] defined a subset of changes in risk, which is in the intersection of MPIR and CD, termed strong risk dominance.

Definition $4.8 X$ is less risky then $Y$ in the sense of strong risk dominance if $Y$ MPIR $X$ and the difference of their distribution functions, $F_{X}(x)-F_{Y}(x)$ is non-decreasing on $\left(a_{1}, b_{1}\right)$, where $a_{2} \leq a_{1}<0<b_{1} \leq b_{2}$, Supp $F_{X}$ is contained in $\left[a_{1}, b_{1}\right]$, and Supp $F_{Y}$ is contained in $\left[a_{2}, b_{2}\right]$.

"The property which characterizes this category of mean preserving spread is that a strong increase in risk is carried out by transferring probability mass from locations, to points at or to the left and to the right of the endpoints of the interval over which the less risky distribution is defined."

Example:

Let $a_{1}=-1, a_{2}=-2, b_{1}=2$, and $b_{2}=3$

Let $F_{X}$ and $F_{Y}$ be two uniform distribution functions defined by $F_{X}(x)=\frac{x+1}{3}$, and $F_{Y}(x)=\frac{x+2}{5}$. Let $f_{X}, f_{Y}$ be respectively the density functions of $F_{X}, F_{Y}$, defined by: $f_{X}(x)=\frac{1}{3} 1_{[-1,2]}$ and $f_{Y}(x)=\frac{1}{5} 1_{[-2,3]}$. We have $\int_{-1}^{2} x f_{X}(x) d x=$ $\int_{-2}^{3} x f_{Y}(x) d x=\frac{1}{2}$, and $\int_{-2}^{y}\left[F_{X}(x)-F_{Y}(x)\right] d x=\frac{1}{15}(y-3)(y+2) \leq 0$, for all $y \in[-2,3]$. So $X$ is less risky than $Y$ in the sense of strong risk dominance. 
Proposition 4.2 If $X$ is less risky than $Y$ for the strong risk dominance, then $X \succeq_{R S D} Y$.

Proof

Let $X, Y \in \mathbb{V}^{+}$such that: $X$ is less risky than $Y$ in the sense of strong risk dominance. Let $\left[a_{1}, b_{1}\right]$ and $\left[a_{2}, b_{2}\right]$, where $a_{2} \leq a_{1}<0<b_{1} \leq b_{2}$, be respectively the supports of $F_{X}$ and $F_{Y}$ and define $H(x)=F_{X}(x)-F_{Y}(x)$.

Let us consider the two possible cases:

i) $H\left(a_{1}\right) \geq 0$ : We have $\forall t<a_{1}, H(t) \leq 0$. Since $H($.$) is non-decreasing in$ $\left(a_{1}, b_{1}\right), H(t) \geq 0, \forall t \geq a_{1}$ and $H($.$) is non-decreasing in \left[a_{1}, 0\right)$.

ii) $H\left(a_{1}\right)<0$ : We have that $H\left(b_{1}\right)=F_{X}\left(b_{1}\right)-F_{Y}\left(b_{1}\right)=F_{Y}\left(b_{1}\right)-1 \geq$

0 . Since $H$ is non-decreasing in $\left(a_{1}, b_{1}\right)$, there exists $t_{0} \in\left(a_{1}, b_{1}\right]$ such that $H(t) \leq 0, \forall t<t_{0}, H(t) \geq 0, \forall t \geq t_{0}$ and if $t_{0}>0, H(t)$ is non-decreasing in $\left[0, t_{0}\right)$, if $t_{0}<0, H(t)$ is non-decreasing in $\left[t_{0}, 0\right)$.

\subsubsection{Simple Dominance}

Let us recall the definition of simple dominance introduced by Dionne and Gollier [2]:

Definition 4.9 Let $X, Y \in \mathbb{V}^{+}, X$ is less risky than $Y$ in the sense of simple dominance if: $E(X)=E(Y), F_{X}(t) \leq F_{Y}(t), \forall t<0$ and $F_{X}(t) \geq F_{Y}(t), \forall t \geq$ 0 .

This definition simply says that $F_{Y}$ crosses $F_{X}$ at zero. $Y$ MPS $X$.

Hence simple dominance is the particular case of relative simple dominance, when $t_{0}=0$.

Quiggin [9] gives an alternative notion of stochastic dominance: the monotone spread, this definition say that the riskier variable should have more weight in the tails, and that the riskier random variable is derived by adding an additional random variable which is co-monotone with the less risky one.

Definition 4.10 Two random variables $X_{1}$ and $X_{2}$ are comonotone if $\forall s, s^{\prime} \in$ $S$,

$\left(X_{1}(s)-X_{1}\left(s^{\prime}\right)\right)\left(X_{2}(s)-X_{2}\left(s^{\prime}\right)\right) \geq 0$.

Let us gives the definition of a particular case of monotone spread introduced by Quiggin [10] called monotone spread about the origin:

Definition 4.11 $Y$ is a mean preserving monotone spread about the origin of $X$, if there exists a mean-zero random variable $Z$ such that $X$ and $Z$ are comonotone, $Y$ has the same probability distribution as $X+Z$ and $X . Z \geq 0 . X$ is said to be less risky than $Y$ in the sense of monotone risk dominance about the origin.

Proposition 4.3 Let $X, Y \in \mathbb{V}^{+}$, if $X$ is less risky than $Y$ for the monotone risk dominance about the origin, then $X$ is less risky than $Y$ in the sense of simple dominance. 
$X, Y \in \mathbb{V}^{+}$such that $Y={ }_{d} X+Z, X$ and $Z$ are comonotone and $X . Z \geq 0$. If $t<0$ :

$P(Y \leq t)=P(X+Z \leq t)$. Since $X . Z \geq 0, t<0$ implies $\{X \leq t\} \subset$ $\{X+Z \leq t\}$, therefore $P(X \leq t) \leq P(Y \leq t)$. That is $F_{X}(t) \leq F_{Y}(t), \forall t<0$. If $t>0$ :

$P(Y>t)=P(X+Z>t)$. Since $X . Z \geq 0, t>0$ implies $\{X>t\} \subset$ $\{X+Z>t\}$, therefore $P(X>t) \leq P(Y>t)$. Thus $F_{X}(t) \geq F_{Y}(t), \forall t>0$.

Right continuity of $F_{X}$ and $F_{Y}$, therefore implies $F_{X}(t) \geq F_{Y}(t), \forall t \geq 0$, which completes the proof.

Remark 4.4 Note that the proof of Proposition 4.3 does not need that $X$ and $Z$ be comonotone. In other words, if $X \in \mathbb{V}^{+}$and $Y$ has the same probability distribution as $X+Z$ and $X . Z \leq 0$, then $X$ is less risky than $Y$ in the sense of simple dominance, accordingly $X \succeq_{R S D} Y$.

\section{Conclusion}

In this paper, we introduce a new class of dominance, termed relative dominance, that we prove to be consistent both with mean preserving in risk and central dominance for $m=1$. Accordingly, in the standard portfolio problem, any increase in risk of the risky asset in the sense of relative dominance will be considered as an actual increase in risk by a risk averse EU decision maker, and moreover this agent will decrease his demand for the risky asset after such a shift.

It is an open question left for future research to know if in fact relative dominance characterizes exactly consistency with both mean preserving in risk and central dominance for $m=1$.

\section{Appendix}

Let us prove lemma 4.1:

Proof

Let $G$ and $H$ are two functions of bounded variations over the interval $[a, b] \subset \mathbb{R}$ and $E=\left\{\left(t, t^{\prime}\right), a \leq t \leq b, a \leq t^{\prime} \leq t\right\}$. Then define $E_{t}=\left\{t^{\prime} \in E, a \leq t^{\prime} \leq t\right\}$ and $E_{t^{\prime}}=\left\{t \in E, t^{\prime} \leq t \leq b\right\}$. From the Fubini's theorem, we have:

$$
\begin{gathered}
\int_{[a, b]}\left[\int_{E_{t}} d H\left(t^{\prime}\right)\right] d G(t)=\int_{[a, b]}\left[\int_{E_{t^{\prime}}} d G(t)\right] d H\left(t^{\prime}\right) \\
\int_{[a, b]}\left[\int_{[a, t]} d H\left(t^{\prime}\right)\right] d G(t)=\int_{[a, b]}\left[\int_{\left[t^{\prime}, b\right]} d G(t)\right] d H\left(t^{\prime}\right)
\end{gathered}
$$




$$
\begin{aligned}
& \int_{[a, b]}\left[H\left(t^{+}\right)-H\left(a^{-}\right)\right] d G(t)=\int_{[a, b]}\left[G\left(b^{+}\right)-G\left(t^{\prime}-\right)\right] d H\left(t^{\prime}\right) \\
& -H\left(a^{-}\right) G\left(b^{+}\right)+H\left(a^{-}\right) G\left(a^{-}\right)+\int_{[a, b]} H\left(t^{+}\right) d G(t)=G\left(b^{+}\right) H\left(b^{+}\right)-G\left(b^{+}\right) H\left(a^{-}\right) \\
& -\int_{[a, b]} G\left(t^{\prime}\right) d H\left(t^{\prime}\right)
\end{aligned}
$$

Hence

$$
\int_{[a, b]} H\left(t^{+}\right) d G(t)+\int_{[a, b]} G\left(t^{-}\right) d H(t)=G\left(b^{+}\right) H\left(b^{+}\right)-H\left(a^{-}\right) G\left(a^{-}\right) .
$$

If $G(t)=t$ and $H$ is right continuous (i.e. $H\left(t^{+}\right)=H(t)$ ), we obtain:

$$
\int_{[a, b]} H(t) d t+\int_{[a, b]} t d H(t)=b H(b)-a H\left(a^{-}\right) .
$$

\section{References}

[1] Cohen, M. (1995): Risk Aversion Concepts in Expected and NonExpected Utility Models. Geneva Papers on Risk and Insurance Theory, 20.

[2] Diamond, P. and Stiglitz, J-E. (1974): Increases in Risk and Risk Aversion. Journal of Economic Theory, 8, 337-360.

[3] Dionne, G. and C.Gollier (1992): Comparative statics under Multiple sources of Risk with Applications to insurance Demand. The Geneva Papers on Risk and Insurance Theory, 17, 21-33.

[4] Dionne, G. Eeckhoudt, L. and Gollier, C. (1993): Increases in risk and linear payoffs. International Economic Review, 34 309-319.

[5] Gollier, C. (1995): The Comparative Statics of Changes in Risk Revisited. Journal o Economic Theory, 66, 522-533.

[6] Machina, M-J. and Pratt, J-W. (1997): Incresing Risk: Some Direct Constructions. Journal of Risk and Uncertainty, 14-15, 103-127.

[7] Meyer, J. and Ormiston,M. (1983): The Comparative Statics of Cumulative Distribution Function Changes for the class of Risk Averse Agents. Journal of Economic Theory, 31, 153-169.

[8] Meyer, J. and Ormiston, M. (1985): Strong Increases in Risk and their Comparative Statics. International Economic Review, 26, 425-437.

[9] Quiggin, J. (1991): Compartive Statics of Rank Dependant Expected Utility Thoery. Journal of Risk and Uncertainty, 4, 339-350. 
[10] Quiggin, J. (1992): Increasing Risk - Another Definition. In A.Chikan (Ed.), Progress in Decision, Utility and Risk Theory, Kluwer, Dordrecht.

[11] Rothschild, M. and Stiglitz, J-E. (1970): Increasing Risk: I.A Definition. Journal of Economic Theory, 2, 225-243.

[12] Rothschild, M. and Stiglitz, J-E. (1971): Increasing Risk: II. Its Economic Consequences. Journal of Economic Theory, 3, 66-84. 